\title{
Influence of osteopathic manipulation on blood flow velocity of the cerebral circulation in chronic mechanical neck pain*
}

\author{
Influência da manipulação osteopática na velocidade de fluxo sanguíneo da circulação cerebral \\ em indivíduos com cervicalgia mecânica crônica
}

\author{
Rafael Stelle ${ }^{1}$, Bianca Simone Zeigelboim¹, Marcos C. Lange², Jair Mendes Marques \\ ${ }^{*}$ Recebido da Universidade Tuiuti do Paraná e Hospital de Clínicas da Universidade Federal do Paraná, Curitiba, PR, Brasil.
}

\section{ABSTRACT}

BACKGROUND AND OBJECTIVES: Osteopathic manipulation is indicated for pain, myofascial tensions and/or decreased movement amplitude. This study aimed at checking whether osteopathic manipulation with cervical rhythmic articulatory technique generates abnormal blood flow velocity oscillations or risks to internal carotid, vertebral and basilar arteries circulation.

METHODS: The sample was made up of 58 individuals with chronic mechanical cervical pain (40 females and 18 males), with mean age of 36 years, submitted to internal carotid, vertebral and basilar arteries ultrasound before and after a single osteopathic manipulation with cervical rhythmic articulatory technique. Individuals were evaluated by ultrasound in three moments: control evaluation, rest control evaluation and study evaluation. Separation was sequential and methods were randomly and blindly applied.

RESULTS: Ultrasound has shown no significant differences in the comparison of flow velocity variables means among evaluations. However, a slight increase in vertebral, intracranial and basilar arteries blood flow was observed after osteopathic manipulation with cervical rhythmic articulatory technique in the study evaluation, without statistical significance.

CONCLUSION: In this studied population, osteopathic manipulation with cervical rhythmic articulatory technique has not generated significant blood flow velocity oscillation of internal carotid, vertebral and basilar arteries and has not posed risk to brain circulation. Keywords: Carotid arteries, Cervical pain, Doppler ultrasound, Spine manipulation, Vertebral artery.

1. Universidade Tuiuti do Paraná, Curitiba, PR, Brasil.

2. Universidade Federal do Paraná, Hospital de Clínicas, Departamento de Neurologia, Curitiba, PR, Brasil.

Apresentado em 30 de junho de 2014.

Aceito para publicaçáo em 03 de novembro de 2014.

Conflito de interesses: não há - Fontes de fomento: năo há.

Endereço para correspondência:

Rafael Stelle

Rua Camôes, 1825 - Bairro Hugo Lange

80040-180 Curitiba, PR, Brasil.

E-mail: osteocuritiba@gmail.com

(c) Sociedade Brasileira para o Estudo da Dor

\section{RESUMO}

JUSTIFICATIVA E OBJETIVOS: Na presença de dor, tensôes miofasciais e/ou redução da amplitude de movimento, é indicada a manipulação osteopática. $\mathrm{O}$ objetivo deste estudo foi verificar se a manipulação osteopática com técnica articulatória rítmica cervical gera oscilaçôes anormais de velocidade de fluxo sanguíneo ou riscos à circulação das artérias carótidas internas, vertebrais e basilar.

MÉTODOS: A casuística foi constituída por 58 indivíduos com cervicalgia mecânica crônica (40 mulheres, 18 homens) com idade média de 36 anos, submetidos a ultrassonografia das artérias carótidas internas, vertebrais e basilar antes e após única manipulação osteopática com técnica articulatória rítmica cervical. Os indivíduos foram analisados pela ultrassonografia em três momentos: exame controle, exame controle de repouso e exame estudo. A separação se deu de forma sequencial e os métodos de forma randomizada e encoberta.

RESULTADOS: A ultrassonografia demonstrou que não existe diferença significativa em nenhum dos casos analisados, no comparativo das médias das variáveis de velocidade de fluxo entre os exames. Porém, foi observado um discreto aumento na velocidade de fluxo sanguíneo das artérias vertebral, intracraniana e basilar, após a manipulação osteopática com técnica articulatória rítmica cervical no exame estudo sem significância estatística.

CONCLUSÁO: Nessa população estudada, a manipulação osteopática com técnica articulatória rítmica cervical não gerou significativa oscilação da velocidade de fluxo sanguíneo das artérias carótidas internas, vertebrais e basilar e não ofereceu risco à circulação cerebral. Descritores: Artérias carótidas, Artéria vertebral, Cervicalgia, Manipulação da coluna, Ultrassonografia Doppler.

\section{INTRODUÇÃO}

As disfunções somáticas vertebrais e as lesóes de coluna são geralmente causadas por movimento brusco e inesperado. Essas disfunçóes geram uma sensibilização do circuito neural medular, periférico e autônomo, chamado de fenômeno de sensibilização ou facilitação medular, onde há uma hiperatividade simpática com aumento do tônus vascular, congestão venosa e linfática, alteração dos reflexos víscero-somáticos e tensóes miofasciais. Com isso podem surgir alguns sinais e sintomas como cervicalgia, alteração postural e redução da amplitude de alguns movimentos ${ }^{1-8}$. Em indivíduos normais, apesar do complexo trajeto, o fluxo sanguíneo das artérias vertebrais 
(AV) não deve ser prejudicado por movimentos normais da coluna cervical, pois ocorre compensaçáo imediata e suficiente da irrigaçáo arterial para o encéfalo através dos ramos e comunicaçóes arteriais. No caso de insuficiência vertebrobasilar (IVB), os testes clínicos envolvem a extensão cervical associada à rotação superior a $45^{\circ}$ ou $50^{\circ}$ que impóe uma compressão da artéria vertebral. Durante a realização desses testes pode ocorrer sintomas típicos como tontura ou vertigem, transtornos visuais, nistagmo ou até desmaio, mas raramente causam acidente vascular encefálico ou morte ${ }^{9-13}$. No entanto, tais sinais e sintomas também podem sugerir vertigem posicional paroxística benigna, e não $\mathrm{IVB}^{14}$.

A manipulação osteopática (MO) tem como objetivo tratar as disfunçôes somáticas ou hipomobilidades vertebrais que estão correlacionadas a dor, tensóes miofasciais, perda da amplitude de movimento, alteração postural, tontura de origem cervical, algumas cefaleias etc. ${ }^{7,8,11,12,15,16}$. Após a manipulação ou mobilização cervical considera-se que há um efeito normalizador sobre o sistema nervoso, permitindo uma normalizaçáo do tônus (espasmo) vascular, bem como nas artérias vertebrais e carótidas, com melhora da velocidade do fluxo sanguíneo por análise de ultrassonografia ${ }^{12,13}$, melhora da força e resistência muscular ${ }^{15}$ e redução da dor de cabeça ${ }^{16}$. São diversas as técnicas osteopáticas, e entre elas está a técnica articulatória rítmica cervical (TARC) $)^{3,7,8,12}$. É descrito que a dissecção da AV associada à manipulaçáo cervical é rara, mas pode ser grave ou fatal em alguns $\operatorname{casos}^{17-19}$. Há descriçôes de que a manipulação e mobilização cervical não acometem risco às AV e carótidas ${ }^{13,20-22}$, e é considerado que a dissecção ou lesão das artérias vertebrais e carótidas deve ser atribuída ao impacto mecânico comum em acidentes automobilísticos (whiplash) ou às doenças arteriais, sendo raro que a manipulação cervical gere tal lesão $\mathrm{o}^{13,19,21,23}$.

A ultrassonografia vascular é indicada para examinar o fluxo sanguíneo das artérias carótidas internas (ACI), vertebrais (AV) e basilar $(\mathrm{AB})$. Esse exame fornece de forma não invasiva o rastreio das artérias, que analisa a integridade dos sistemas vertebrobasilar ou carotídeos, através da velocidade de fluxo arterial entre outros dados ${ }^{12,24,25}$. O objetivo deste estudo foi verificar se a MO-TARC gera oscilaçóes anormais ou riscos à circulação das artérias carótidas internas, vertebrais e basilar. A hipótese é de que a MO-TARC pode gerar oscilaçôes no fluxo sanguíneo constatada pela ultrassonografia sem prejudicar a saúde. Será a MO-TARC uma técnica terapêutica segura para a saúde dessas artérias em indivíduos com cervicalgia mecânica?

\section{MÉTODOS}

A casuística foi constituída de 58 indivíduos com cervicalgia mecânica crônica, sendo 18 homens e 40 mulheres, com idade média de $36,0 \pm 6,5$ anos, (homens: $36,5 \pm 6,1$ anos / mulheres: 34,8 \pm 7,3 anos), envolvendo um grupo voluntário de funcionários do Hospital de Clínicas da Universidade Federal do Paraná. O período do estudo foi de agosto de 2010 a março de 2012.

Os critérios de inclusão foram indivíduos com cervicalgia comum crônica de origem mecânica, de intensidade leve e moderada conforme o Índice de Incapacidade Relacionada ao Pescoço (Neck Disability Index), na faixa etária de 25 a 45 anos de ambos os gêneros. Os critérios de exclusão foram qualquer alteração que impedisse a realização do protocolo, como dor intensa ou incapacitante, tontura ou vertigem e outros sinais e sintomas de IVB, alteração de fluxo arterial na primeira ultrassonografia do protocolo, hipomobilidade cervical grave (p. ex. uncoartrose, discopatia, malformação óssea), deformidade de coluna (p. ex. doença de Scheuermann), indivíduos em estado pós-cirúrgico, sequela por trauma de crânio ou coluna, em uso de muletas, andador ou cadeira de rodas.

Os indivíduos foram analisados pela ultrassonografia em três momentos (das ACIs, $\mathrm{AVs}$ e $\mathrm{AB}$ ), incluindo exame controle (EC), exame controle do repouso de 5 min (ECR) e exame estudo (EE). A separação foi de forma sequencial e os métodos de forma randomizada e encoberta. Os procedimentos foram realizados em uma única sessão de aproximadamente $30 \mathrm{~min}$. Após a entrevista, coleta de dos dados e assinatura do Termo de Consentimento Informado Livre e Esclarecido (TCLE), o indivíduo deitou-se em decúbito dorsal sobre uma maca e travesseiro pequeno e baixo (tipo infantil), permanecendo assim em ambiente silencioso até o final da seguinte sequência de procedimentos: (1) Ultrassonografia (EC); (2) Repouso ou MO-TARC; (3) Ultrassonografia (ECR ou EE); (4) MO-TARC ou Repouso; (5) Ultrassonografia (ECR ou EE). Em uma sequência aleatória, dos 58 indivíduos, 29 realizaram primeiro o Método Repouso e depois o método MO-TARC, e os outros 29 indivíduos realizaram primeiramente o método MO-TARC e depois o método Repouso. Essa separação foi aplicada no caso de possibilidade de diferenças nos resultados. Os métodos foram realizados sempre pelos mesmos profissionais (operador), sendo o operador-1 para a ultrassonografia e o operador-2 para o controle do repouso e execução da MO-TARC.

\section{Ultrassonografia vascular}

Esses exames foram realizados pelo mesmo examinador cego (operador-1), para o momento da avaliação, com aparelho de ultrassom modelo VIVID E, marca GE, com transdutor linear de 7,5 a 10 $\mathrm{MHz}$ para circulação extracraniana, e com transdutor transversal de 1,5 a $5 \mathrm{MHz}$ para circulação intracraniana. Foram realizadas três etapas de exame (EC, ECR, EE), de 3 min cada, onde logo após cada uma o operador-1 ausentava-se da sala e após 5 minutos retornava para a próxima etapa. Após o exame de rotina para avaliação de achados anormais em carótidas e vertebrais, e ausência de alteraçóes patológicas, foram salvas amostras do Doppler arterial para as artérias ACI direita (ACID), ACI esquerda (ACIE), AV direita (AVD) e AV esquerda (AVE) (Figura 1), AVD em seu segmento intracraniano (AVDintra), AVE em seu segmento intracraniano (AVEintra) e $A B$. As segunda e terceira etapas foram iniciadas com análise direta dos vasos ACIE, AVE, ACID, AVD, AVEintra, AVDintra e AB. Em todos os vasos analisados foram coletadas as seguintes variáveis: velocidade de pico sistólico (VPS); velocidade diastólica final (VDF); velocidade média (VM); índice de pulsatilidade (IP); índice de resistência (IR). Os três últimos foram coletados por meio de fórmula.

\section{MO-TARC}

A MO-TARC foi realizada através das mãos do operador-2, envolvendo o pescoço do sujeito com os indicadores próximos de cada vértebra e sua articulação interfacetária (regiẫo posterior dos processos transversos). Executando movimentos passivos de forma rítmica e suave com 3 repetiçóes para cada articulação interfacetária 


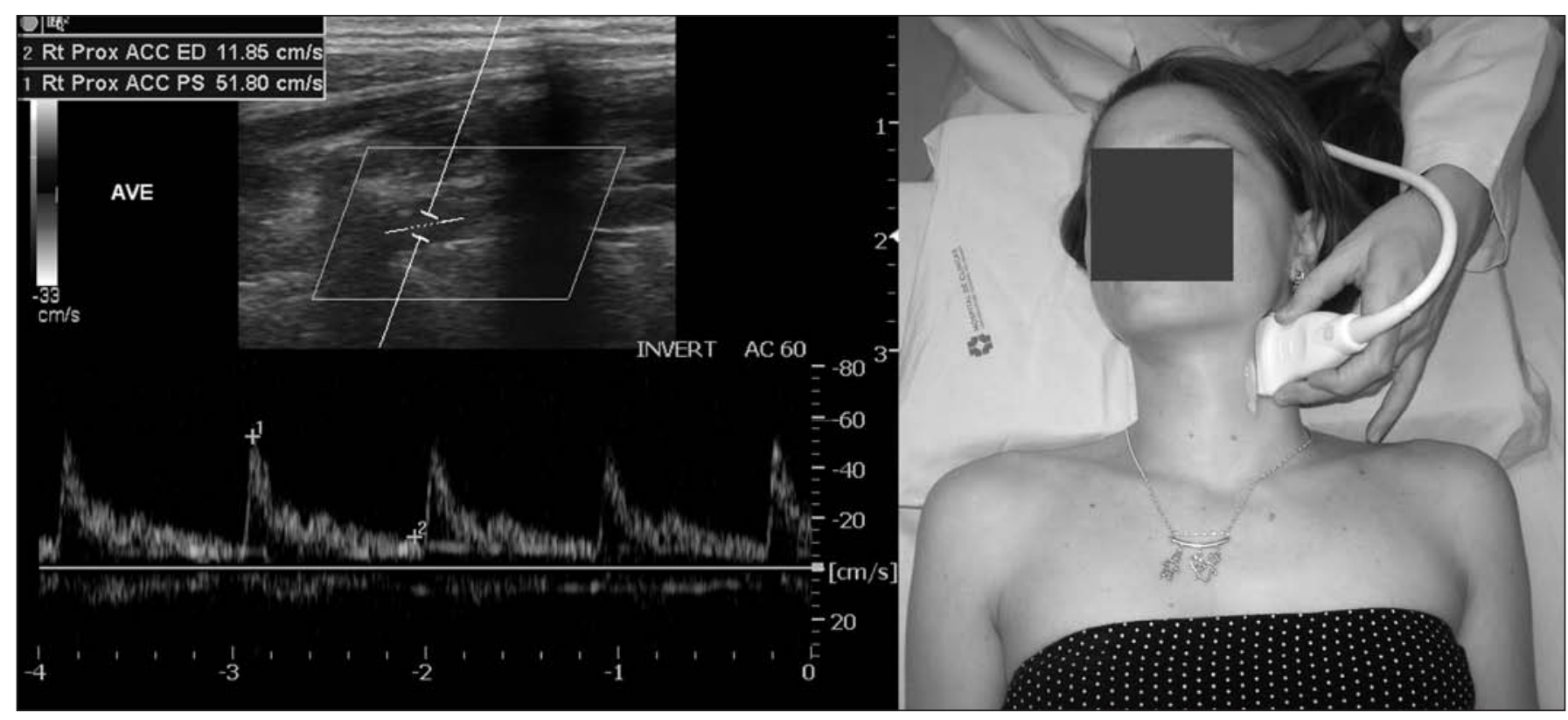

Figura 1. Ultrassonografia arterial: imagem do vaso sanguíneo e imagem do efeito Doppler do fluxo sanguíneo, e aplicação do método

(zigoapofisária), ou seja, com mobilizaçóes de um lado para o outro, associando deslizamento lateral com rotação (Figura 2A), formando movimento em " $\infty$ " na vista axial. Iniciou-se o processo na primeira vertebra torácica (T1) ascendendo por todas as cervicais até as articulaçóes atlantoccipitais. Na cervical superior foram acrescentadas
3 mobilizaçôes em flexão e 3 em extensão bilateral dos côndilos occipitais (atlantoccipital) (Figura 2B), mais 3 deslizamentos laterais para o atlas (Figura 2C), e 3 rotaçóes para C3 e 3 rotaçóes para C2C1 (Figura 2D). Para as atlantoccipitais, uma das mãos ficava sobre a cabeça do sujeito (regiáo frontal ou lateral).

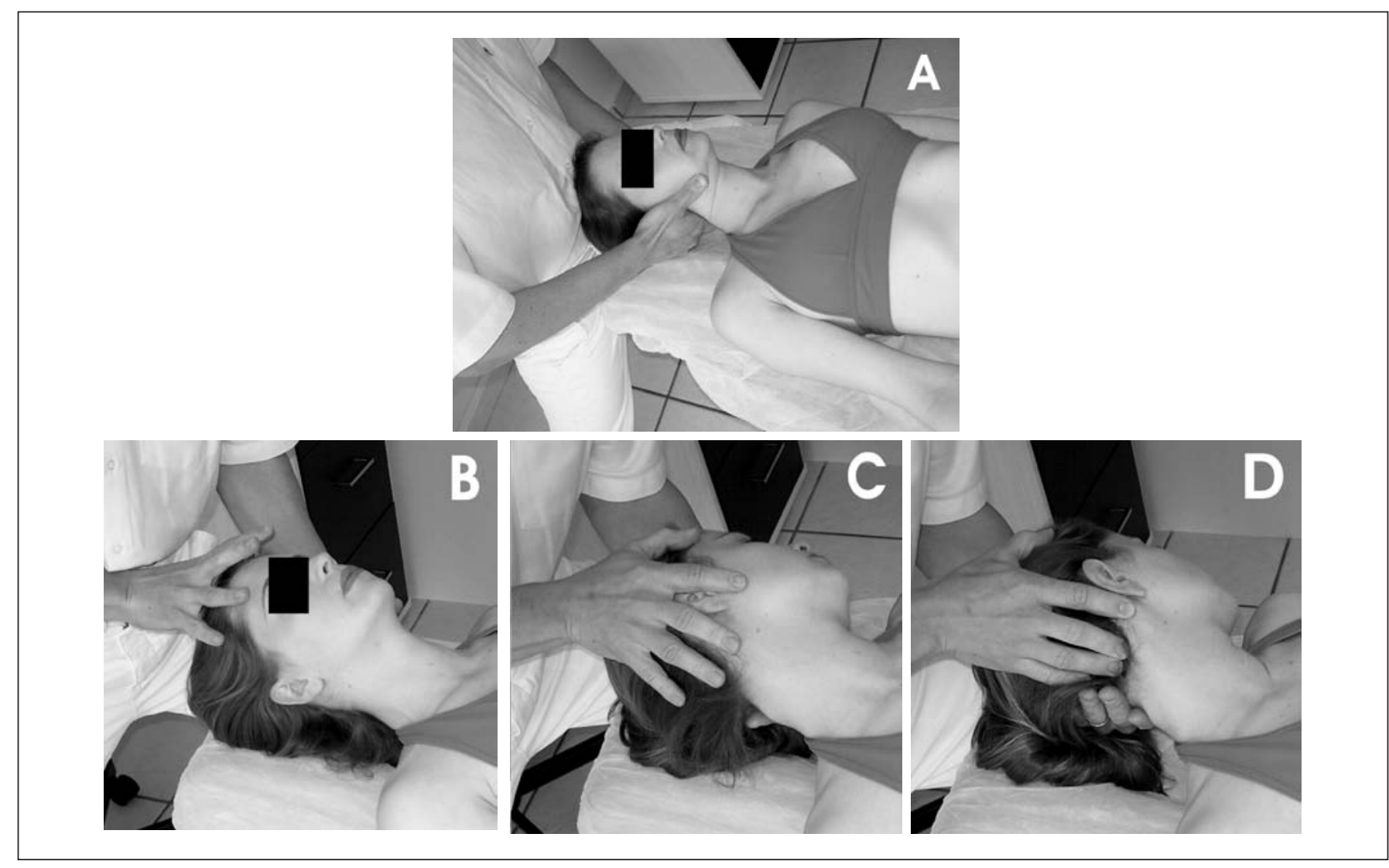

Figura 2. Manipulação osteopática com técnica articulatória rítmica cervical. (A) Deslizamento. (B) Flexão e extensão da atlantoccipital. (C) Deslizamento de atlas. (D) Rotação atlas-axis 


\section{Repouso}

Controlado pelo operador-2, o sujeito foi instruído a relaxar e descansar por 5 minutos.

\section{Análise estatística}

Foi utilizado o teste $t$ de Student, considerando-se o nível de significância de 0,05 (5\%). Logo após o início da pesquisa, incluiu-se no método a ultrassonografia das carótidas, tendo sido analisados 46 indivíduos para artéria carótida interna e 58 indivíduos para artéria vertebral e basilar. Essa diferença não interferiu nos resultados, pois a análise foi perante cada artéria e não um comparativo entre as diferentes artérias, as quais diferem em calibre e fluxo sanguíneo. $\mathrm{O}$ programa utilizado foi Excel 2010.

Este estudo foi aprovado pelo Comitê de Ética em Pesquisa (Registro CEP-HC-UFPR: 2233.127/2010-06) e está em concordância com a Declaração de Helsinque.

\section{RESULTADOS}

A tabela 1 demonstra que não existiu diferença significativa em nenhum dos casos analisados no comparativo das médias das variáveis de velocidade de fluxo (VPS, VDF, VM) entre EC e EE, com a apli- cação do teste $t$ de Student ao nível de significância de 0,05 (5\%). Também se pode verificar na tabela 1 , porém sem significância estatística, um discreto aumento da velocidade de fluxo nas artérias intracranianas, mais notável na vertebral intracraniana direita, com VPS de 48,86 para $50,77 \mathrm{~cm} / \mathrm{seg}$; VDF de 23,74 para $25,26 \mathrm{~cm} /$ seg; VM 32,11 para 33,77 cm/seg. Para reduzir o tamanho das tabelas, foi excluído o índice de pulsatilidade e o índice resistência.

A tabela 2 demonstra que não existiu diferença significativa entre o comparativo das médias de velocidade de fluxo (VPS, VDF, VM) entre o ECR e EE, com a aplicação do teste $t$ de Student, ao nível de significância de 0,05 (5\%). Pode-se verificar na tabela, porém sem significância estatística, um discreto aumento da velocidade de fluxo nas artérias intracranianas, mais notável na vertebral intracraniana direita, com VPS de 47,56 para 50,77 cm/seg; VDF de 23,21 para $25,26 \mathrm{~cm} / \mathrm{seg}$; VM de 31,33 para $33,77 \mathrm{~cm} / \mathrm{seg}$.

A tabela 3 mostra que existiu diferença significativa entre o comparativo das médias de velocidade de fluxo (VPS e VM) entre o EC e ECR na artéria carótida direita, pela aplicação do teste $t$ de Student, ao nível de significância de 0,05 (5\%). Considerou-se sem significância estatística, uma discreta redução da velocidade de fluxo (VPS, VDF e VM) nas outras artérias, e na VDF da artéria carótida interna direita.

Tabela 1. Comparação entre as médias dos entre exames controle e estudo (exame 1 e exame pós-MO-TARC)

\begin{tabular}{|c|c|c|c|c|c|}
\hline Vaso e lado & Variáveis & $\begin{array}{c}\text { Média } \\
\text { EC(cm/seg) }\end{array}$ & $\begin{array}{c}\text { Média } \\
\mathrm{EE}(\mathrm{cm} / \mathrm{seg})\end{array}$ & $\begin{array}{c}\text { Estatística } t \text { de } \\
\text { Student }\end{array}$ & Valor de $p$ \\
\hline \multirow[t]{3}{*}{ Vert. extra D } & VPS & 51,69 & 46,98 & 1,540 & 0,1263 \\
\hline & VDF & 15.86 & 15,05 & 0,721 & 0,4722 \\
\hline & VM & 28,05 & 25,65 & 1,432 & 0,1550 \\
\hline \multirow[t]{3}{*}{ Vert. extra E } & VPS & 53,48 & 50,28 & 1,318 & 0,1900 \\
\hline & VDF & 17,03 & 16,47 & 0,545 & 0,5870 \\
\hline & VM & 29,18 & 27,74 & 1,056 & 0,2934 \\
\hline \multirow[t]{3}{*}{ Vert. intra D } & VPS & 48,86 & 50,77 & $-0,806$ & 0,4219 \\
\hline & VDF & 23,74 & 25,26 & $-1,331$ & 0,1860 \\
\hline & VM & 32,11 & 33,77 & $-1,103$ & 0,2722 \\
\hline \multirow[t]{3}{*}{ Vert. intra E } & VPS & 56,26 & 54,95 & 0,516 & 0,6067 \\
\hline & VDF & 26,83 & 27,02 & $-0,156$ & 0,8765 \\
\hline & VM & 36,64 & 36,33 & 0,195 & 0,8459 \\
\hline \multirow[t]{3}{*}{ Basilar } & VPS & 64,17 & 65,31 & $-0,430$ & 0,6679 \\
\hline & VDF & 29,69 & 31,10 & $-1,141$ & 0,2564 \\
\hline & VM & 39,51 & 40,47 & $-0,507$ & 0,6133 \\
\hline \multirow[t]{3}{*}{ Carótida D } & VPS & 81,22 & 77,20 & 0,847 & 0,3994 \\
\hline & VDF & 29,22 & 27,63 & 0,858 & 0,3934 \\
\hline & VM & 46,55 & 44,15 & 0,987 & 0,3264 \\
\hline \multirow[t]{3}{*}{ Carótida E } & VPS & 83,04 & 80,13 & 0,658 & 0,5119 \\
\hline & VDF & 31,11 & 30,43 & 0,404 & 0,6870 \\
\hline & VM & 48,42 & 47,00 & 0,608 & 0,5445 \\
\hline
\end{tabular}

D: direito; E: esquerdo; Vert. extra: vertebral extracraniana; Vert. intra: vertebral intracraniana; EC: exame controle; EE: exame estudo; VPS: velocidade de pico sistólico; VDF: velocidade diastólica final; VM: velocidade média. 
Tabela 2. Comparação entre as médias dos exames controle-repouso e estudo (exame pós-repouso e exame pós MO-TARC)

\begin{tabular}{|c|c|c|c|c|c|}
\hline Vaso e lado & Variáveis & $\begin{array}{c}\text { Média } \\
\text { ECR }(\mathrm{cm} / \mathrm{seg})\end{array}$ & $\begin{array}{c}\text { Média } \\
\text { EE(cm/seg) }\end{array}$ & $\begin{array}{c}\text { Estatística } t \text { de } \\
\text { Student }\end{array}$ & Valor de $p$ \\
\hline \multirow[t]{3}{*}{ Vert. extra D } & VPS & 48,36 & 46,98 & 0,448 & 0,6548 \\
\hline & VDF & 15,67 & 15,05 & 0,525 & 0,6005 \\
\hline & VM & 26,97 & 25,65 & 0,758 & 0,4497 \\
\hline \multirow[t]{2}{*}{ Vert. extra $\mathrm{E}$} & VPS & 51,60 & 50,28 & 0,463 & 0,6444 \\
\hline & VDF & 16,62 & 16,47 & 0,144 & 0,8860 \\
\hline \multirow[t]{3}{*}{ Vert. intra D } & VPS & 47,56 & 50,77 & $-1,330$ & 0,1863 \\
\hline & VDF & 23,21 & 25,26 & $-1,768$ & 0,0797 \\
\hline & VM & 31,33 & 33,77 & $-1,588$ & 0,1151 \\
\hline \multirow[t]{2}{*}{ Vert. intra E } & VPS & 54,14 & 54,95 & $-0,316$ & 0,7528 \\
\hline & VDF & 26,41 & 27,02 & $-0,429$ & 0,6691 \\
\hline Basilar & VM & 39,29 & 40,47 & $-0,578$ & 0,5645 \\
\hline \multirow[t]{3}{*}{ Carótida D } & VPS & 71,91 & 77,20 & $-1,236$ & 0,2198 \\
\hline & VDF & 27,02 & 27,63 & $-0,330$ & 0,7424 \\
\hline & VM & 41,99 & 44,15 & $-0,954$ & 0,3425 \\
\hline \multirow[t]{3}{*}{ Carótida E } & VPS & 79,54 & 80,13 & $-0,142$ & 0,8871 \\
\hline & VDF & 29,33 & 30,43 & $-0,762$ & 0,4483 \\
\hline & VM & 46,07 & 47,00 & $-0,447$ & 0,6557 \\
\hline
\end{tabular}

D: direito; E: esquerdo; Vert. extra: vertebral extracraniana; Vert. intra: vertebral intracraniana; ECR: exame controle de repouso; EE: exame estudo; VPS: velocidade de pico sistólico; VDF: velocidade diastólica final; VM: velocidade média.

Tabela 3. Comparação entre as médias dos estudos controle e controle-repouso (exame 1 e exame pós-repouso)

\begin{tabular}{|c|c|c|c|c|c|}
\hline Vaso e lado & Variáveis & $\begin{array}{c}\text { Média } \\
\text { EC(cm/seg) }\end{array}$ & $\begin{array}{c}\text { Média } \\
\text { ECR }(\mathrm{cm} / \mathrm{seg})\end{array}$ & $\begin{array}{c}\text { Estatística } t \text { de } \\
\text { Student }\end{array}$ & Valor de $p$ \\
\hline \multirow[t]{3}{*}{ Vert. extra D } & VPS & 51,69 & 48,36 & 1,038 & 0,3013 \\
\hline & VDF & 15,86 & 15,67 & 0,159 & 0,8743 \\
\hline & VM & 28,05 & 26,97 & 0,592 & 0,5549 \\
\hline \multirow[t]{3}{*}{ Vert. extra $\mathrm{E}$} & VPS & 53,48 & 51,60 & 0,693 & 0,4899 \\
\hline & VDF & 17,03 & 16,62 & 0,387 & 0,6995 \\
\hline & VM & 29,18 & 28,28 & 0,617 & 0,5382 \\
\hline \multirow[t]{3}{*}{ Vert. intra D } & VPS & 48,86 & 47,56 & 0,562 & 0,5756 \\
\hline & VDF & 23,74 & 23,21 & 0,494 & 0,6220 \\
\hline & VM & 32,11 & 31,33 & 0,545 & 0,5869 \\
\hline \multirow[t]{3}{*}{ Vert. intra E } & VPS & 56,26 & 54,14 & 0,827 & 0,4101 \\
\hline & VDF & 26,83 & 26,41 & 0,289 & 0,7734 \\
\hline & VM & 36,64 & 35,66 & 0,562 & 0,5750 \\
\hline \multirow[t]{3}{*}{ Basilar } & VPS & 64,17 & 64,03 & 0,051 & 0,9597 \\
\hline & VDF & 29,69 & 29,91 & $-0,174$ & 0,8618 \\
\hline & VM & 39,51 & 39,29 & 0,114 & 0,9091 \\
\hline \multirow[t]{3}{*}{ Carótida D } & VPS & 81,22 & 71,91 & 2,182 & ${ }^{*} 0,0317$ \\
\hline & VDF & 29,22 & 27,02 & 1,382 & 0,1703 \\
\hline & VM & 46,55 & 41,99 & 2,147 & ${ }^{*} 0,0345$ \\
\hline \multirow[t]{3}{*}{ Carótida E } & VPS & 83,04 & 79,54 & 0,871 & 0,3862 \\
\hline & VDF & 31,11 & 29,33 & 1,057 & 0,2935 \\
\hline & VM & 48,42 & 46,07 & 1,062 & 0,2909 \\
\hline
\end{tabular}

D: direito; esquerdo: E: lado direito; Vert. extra: vertebral extracraniana; Vert. intra: vertebral intracraniana; EC: estudo controle; ECR: exame controle de repouso; VPS: velocidade de pico sistólico; VDF: velocidade diastólica final; VM: velocidade média. 


\section{DISCUSSÃO}

Nesta pesquisa, a MO-TARC foi executada em todos os sentidos de movimento manipulativo dentro dos limites fisiológicos da coluna cervical, incluindo posicionamentos semelhantes às clássicas manipulaçóes osteopáticas e quiropráticas, sem o uso do posicionamento de extensão com rotação ${ }^{3,7,8,12}$. Sendo assim, os resultados pelo exame imediato de ultrassonografia demonstraram (Tabelas 1 e 2) que não houve oscilaçóes anormais ${ }^{24,25}$ ou significativas na velocidade do fluxo sanguíneo após a MO-TARC na população estudada. É possível afirmar que a MO-TARC em deslizamento e em rotação não gerou risco à circulação de tais artérias, que conforme algumas citaçóes bibliográficas, o tratamento manipulativo osteopático ou a manipulação vertebral não causou lesão ou tensão indevida sobre as artérias vertebrais e carótidas ${ }^{12,13,20-22}$, oferecendo deslizamentos significativamente menores do que os dos testes clínicos para IVB ${ }^{9,10,13,20}$. Portanto, a manipulação cervical, realizada por um profissional, não foi neste estudo um fator de risco ou de lesão vertebrobasilar e carotídea ${ }^{12,13,20-23}$.

Os resultados desta pesquisa confirmam que nenhum indivíduo apresentou IVB ou insuficiência carotídea pela ultrassonografia antes e após a MO-TARC, pois não houve oscilaçôes significativas no fluxo de tais artérias no EC-EE (Tabela 1) e no ECR-EE (Tabela 2), com resultados dentro dos índices de normalidade ${ }^{24,25}$. A pesquisa envolveu indivíduos com cervicalgia comum considerados saudáveis, e comparativamente com as descriçóes citadas, em indivíduos normais o fluxo sanguíneo das artérias vertebrais não deve ser prejudicado por movimentos comuns da coluna cervical ou com a manipulação cervical ${ }^{9,21}$.

Quanto à hipótese de que a MO-TARC poderia aumentar a velocidade do fluxo arterial, ainda não pode ser confirmada, em razão de que, sem significância estatística, houve apenas um discreto aumento da velocidade do fluxo nas artérias intracranianas após MO-TARC (EE: Tabelas 1 e 2). No entanto, se com o repouso houve redução significativa ou discreta da velocidade de fluxo (Tabela 3), então, pode-se afirmar que existe diferença entre executar ou não executar a MO-TARC, o que sugere estudos mais aprofundados em indivíduos com tontura de origem cervical ou com cefaleia.

Após a MO-TARC alguns indivíduos relataram relaxamento muscular ou sensação de conforto corporal. Nenhum dos indivíduos submetidos a MO-TARC ou a ultrassonografia arterial apresentou sintomas álgicos ou outra queixa.

Devido ao prazo e critérios de inclusão e exclusão, finalizou-se a pesquisa com 58 dos 80 indivíduos propostos no anteprojeto.

Apesar de discretas as diferenças no aumento do fluxo, sugerem-se futuras investigaçóes em indivíduos com tontura ou vertigem, onde há possibilidade de significativa oscilação de fluxo.

\section{CONCLUSÃO}

O presente estudo demonstrou que não há significativa oscilação da velocidade de fluxo na artérias vertebrais (bilateral intra e extracranianas), basilar e carótidas internas (bilateral) com a MO-TARC, o que permite oferecer segurança no tratamento manipulativo cervical sem riscos de complicaçóes vasculares conforme observado no grupo estudado.

\section{AGRADECIMENTOS}

A todos os incentivadores e colaboradores desta pesquisa científica, em especial François Ricard, Rogério A. Queiroz, Renato Tambara Filho, Viviana R. Zurro, Maria José Mocelin, Genoveva Freire D’Aquino e minha esposa Karin Teuber Stelle.

\section{REFERÊNCIAS}

1. Bevilagua-Grossi D, Pegoretti KS, Gonçalves MC, Speciali JG, Bordini CA Bigal ME. Cervical mobility in women with migraine. Headache. 2009;49(5):726-31.

2. Armijo Olivo S, Magee DJ, Parfitt M, Major P, Thie NM. The association between the cervical spine, the stomatognathic system, and craniofacial pain: a critical review. J Orofac Pain. 2006;20(4):271-87.

3. Roberge RJ, Roberge MR. Overcoming barriers to the use of osteopathic manipulation techniques in the emergency department. West J Emerg Med. 2009;10(3):184-9.

4. Jasiewics JM, Treleaven J, Condie P, Jull G. Wireless orientation sensors: their suitability to measure head movement for neck pain assessment. Man Ther. 2007;12(4):380-5.

5. Strimpakos N, Sakellari V, Gioftsos G, Papathanasiou M, Brountzos E, Kelekis D, et al. Cervical spine ROM measurements: optimizing the testing protocol by using a $3 \mathrm{D}$ ultrasound-based motion analysis system. Cephalalgia. 2005;25(12):1133-45.

6. Fryer G, Morris T, Gibbons P. Paraspinal muscles and intervertebral dysfunction. J Manipulative Physiol Ther. 2004;27(4):267-74.

7. Stelle R, Zeigelboim BS, Lange MC, Marques JM. Influência da manipulaçâo osteopática na amplitude de rotaçáo da coluna cervical em indivíduos com cervicalgia mecânica crônica. Rev Dor. 2013:14(4):284-9.

8. Mansilla-Ferragut P, Fernández-de-Las-Penās C, Albuquerque-Sendín F, Cleland JA, Boscá-GandíaJJ. Immediate effects of atlanto-occipital joint manipulation on active mouth opening and pressure pain sensitivity in women with mechanical neck pain. J Manipulative Physiol Ther. 2009;32(2):101-6

9. Thomas LC, Rivett DA, Bolton PS. Pre-manipulative testing and the use of the velocimeter. Man Ther. 2008;13(1):29-36.

10. Mitchell J, Keene D, Dyson C, Harvey L, Pruvey C, Phillips R. Is cervical spine rotation, as used in the standard vertebrobasilar insufficiency test, associated with a measureable change in intracranial vertebral artery blood flow? Man Ther. 2004;9(4),220-27.

11. Cleland JA, Mintken PE, Carpenter K, Fritz JM, Glynn P, Whitman J, Childs JD. Examination of a clinical prediction rule to identify patients with neck pain likely to benefit from thoracic spine thrust manipulation and a general cervical range of motion exercise: multi-center randomized clinical trial. Phys Ther.2010;90(9):1239-50.

12. Creighton D, Kondratek M, Krauss J, Huijbregts P, Qu H. Ultrasound analysis of the vertebral artery during non-thrust cervical translatoric spinal manipulation. J Man Manip Ther. 2011;19(2): 85-90.

13. R Pérez-Llanes J, Ríos-Díaz JJ, Martínez-Payá ME, del-Baño-Aledo. Fisioterapia. Science Direct J. 2012;34(3):118-24.

14. Silva AL, Marinho MR, Gouveia FM, Silva JG, Ferreira Ade S, Cal R. [Benign paroxysmal positional vertigo: comparison of two recent international guidelines]. Braz J Otorhinolaryngol. 2011;77(2):191-200. English, Portuguese.

15. Maduro-De-Camargo V, Alburquerque-Sendín F, Bérzin F, Cobos-Stefanelli V, Rodrigues-Pedroni C, Santos K. Immediate effects of the ashmore manipulation technique C5/C6, in muscle activity in patients with mechanical neck pain. Eur J Ost Clin Rel Res. 2012;7(1):2-9.

16. Orelli JG, RebelattoJR. The effectiveness of manual therapy in individuals with headaches, with and without cervical degeneration: analysis of six cases. Rev Bras Fisioter. 2007;11(4):325-9.

17. Leon-Sanchez A, Cuetter A, Ferrer G. Cervical spine manipulation: an alternative medical procedure with potentially fatal complications. South Med J. 2007;100(2):201-3.

18. Chen WL, Chern CH, Wu YL, Lee CH. Vertebral artery dissection and cerebellar infarction following chiropractic manipulation. Emerg Med J. 2006;23(1):e1.

19. Khan AM, Ahmad N, Li X, Korsten MA, Rosman A. Chiropractic sympathectomy: carotid artery dissection with oculosympathetic palsy after chiropractic manipulation of the neck. Mt Sinai J Med. 2005;72(3):207-10.

20. Herzog W, Leonard TR, Symons B, Tang C, WuestS. Vertebral artery strains during high-speed, low amplitude cervical spinal manipulation. J Electromyogr Kinesiol. 2012;22(5):740-6.

21. Haneline M, Triano J. Cervical artery dissection. A comparison of highly dynamic mechanisms: manipulation versus motor vehicle collision. J Manipulative Physiol Ther. 2005;28(1):57-63.

22. Wynd S, Anderson T, Kawchuk G. Effect of cervical spine manipulation on a pre-existing vascular lesion within the canine vertebral artery. Cerebrovasc Dis. 2008;26(3):304-9.

23. Correa E, Martinez B. Traumatic dissection of the internal carotid artery: simultaneous infarct of optic nerve and brain. Clin Case Rep. 2014;2(2):51-6.

24. Barbosa MF, Abdala N, Carrete H Jr, Nogueira RG, Nalli DR, Fonseca JR, et al. [Reference values for measures of blood flow velocities and impedance indexes in healthy individuals through conventional transcranial Doppler]. Arq Neuropsiquiatr. 2006;64(3B):82938. Portuguese.

25. Yazici B, Erdoğmuş B, TugayA. Cerebral blood flow measurements of the extracranial carotic and vertebral arteries with Doppler ultrasonography in healthy adults. Diagn Interv Radiol. 2005;11(4):195-8. 